\title{
Profiling of dynamics in protein-lipid-water systems: a time- resolved fluorescence study of a model membrane protein with the label BADAN at specific membrane depths
}

\author{
Rob B. M. Koehorst - Sergey Laptenok · Bart van Oort · Arie van Hoek • \\ Ruud B. Spruijt · Ivo H. M. van Stokkum • Herbert van Amerongen • \\ Marcus A. Hemminga
}

Received: 29 April 2009/Revised: 19 August 2009/Accepted: 26 August 2009/Published online: 16 September 2009 (C) The Author(s) 2009. This article is published with open access at Springerlink.com

\begin{abstract}
Profiles of lipid-water bilayer dynamics were determined from picosecond time-resolved fluorescence spectra of membrane-embedded BADAN-labeled M13 coat protein. For this purpose, the protein was labeled at seven key positions. This places the label at well-defined locations from the water phase to the center of the hydrophobic acyl chain region of a phospholipid model membrane, providing us with a nanoscale ruler to map membranes. Analysis of the time-resolved fluorescence spectroscopic data provides the characteristic time constant for the twisting motion of the BADAN label, which is sensitive to the local flexibility of the protein-lipid environment. In addition, we obtain information about the mobility of water molecules at the membrane-water interface. The results provide an unprecedented nanoscale profiling of the dynamics and distribution of water in
\end{abstract}

The more you see: spectroscopy in molecular biophysics.

R. B. M. Koehorst - S. Laptenok - B. van Oort - A. van Hoek ·

R. B. Spruijt · H. van Amerongen - M. A. Hemminga

Laboratory of Biophysics, Wageningen University,

Dreijenlaan 3, 6703 HA Wageningen, The Netherlands

R. B. M. Koehorst · A. van Hoek · H. van Amerongen

MicroSpectroscopy Center Wageningen, Dreijenlaan 3,

6703 HA Wageningen, The Netherlands

Present Address:

B. van Oort - I. H. M. van Stokkum

Department of Physics and Astronomy,

VU University Amsterdam, De Boelelaan 1081,

1081 HV Amsterdam, The Netherlands

M. A. Hemminga $(\bowtie)$

Laboratory of Biophysics, Wageningen University,

P.O. Box 8128, 6700 ET Wageningen, The Netherlands

e-mail: marcus.hemminga@wur.nl membrane systems. This information gives clear evidence that the actual barrier of membranes for ions and aqueous solvents is located at the region of carbonyl groups of the acyl chains.

Keywords Membrane-embedded M13 coat protein .

Streak camera picosecond fluorescence .

Dynamic stokes shift $\cdot$ Solvent relaxation .

Membrane polarity $\cdot$ Membrane-bound water

$\begin{array}{ll}\text { Abbreviations } \\ \text { BADAN } & \text { 6-Bromo-acetyl-2-dimethylamino-naphtalene } \\ \text { HB } & \text { Hydrogen bonding } \\ \text { LD } & \text { Label dynamics } \\ \text { PICT } & \begin{array}{l}\text { Intramolecular charge transfer state with planar } \\ \text { conformation }\end{array} \\ \text { SR } & \begin{array}{l}\text { Solvent relaxation } \\ \text { TICT }\end{array} \\ & \begin{array}{l}\text { Intramolecular charge transfer state with } \\ \text { twisted conformation }\end{array}\end{array}$

Introduction

To understand the machinery of life at the molecular level, we need information about the structures, interactions and dynamics of biomolecules and other cellular components. All biomolecular interactions are mediated by the dynamics of the ubiquitous biosolvent water (Persson and Halle 2008). Particularly challenging in this respect is the behavior of molecules at interfaces, especially membranes (Wood et al. 2007). Membranes play a key role in compartmentalizing a biological cell, but also many biological functions are derived from biological membranes. 
Hydration water in the hydrophobic interior of membranes leads to polarity and hydration profiles across it, which are fundamental in maintaining the membrane's architecture as well as in transport and insertion processes (Epand and Kraayenhof 1999; Klymchenko et al. 2004; Sýkora et al. 2007). This work is based on solvent relaxation (Jurkiewicz et al. 2006; Sýkora et al. 2007), i.e., the finding that the excited state of fluorescent probes is sensitive to the ability of the surrounding water molecules to dielectrically stabilize the ground and excited state dipoles of the probe (Lakowicz 2006). In addition, hydration-water dynamics is an important factor for the lipid dynamics and the dynamics of the membrane-embedded proteins (Wood et al. 2007).

Only a few experimental techniques can monitor the structural and dynamical behavior of water molecules at membrane and protein interfaces. Powerful techniques are NMR relaxation experiments that provide information about the state of water over a large dynamic range from picoseconds to even hours (Halle 2004; Persson and Halle 2008) and neutron scattering, which reveals motions on the nano- to picosecond time scale (Jasnin et al. 2008; Wood et al. 2007). Terahertz (THz) spectroscopy is another experimental method that is able to detect water network dynamics at the biomolecule-water interface, and it can report on distance scales beyond current NMR experiments (Ebbinghaus et al. 2008; Ebbinghaus et al. 2007). The analysis of such experimental data is often supplemented by molecular dynamics simulations that explicitly take into account solvent water molecules (Ebbinghaus et al. 2007; Oleinikova et al. 2007; Wood et al. 2007). Recently, the hydration dynamics at a protein surface was mapped out by ultrafast spectroscopy of site-specific introduced tryptophans of various mutants of sperm whale myoglobin (Zhang et al. 2007). This work resulted in two distinct dynamical regimes: one with dynamical timescales of 1-8 ps, the other around 20-200 ps. These regimes are strongly correlated with the protein's structure and composition, confirming an intimate relationship between hydration dynamics and protein fluctuations.

In the past years, we have been using steady-state fluorescence spectroscopy on site-directed fluorescentlabeled M13 coat protein (a reference membrane protein) reconstituted in phospholipid bilayers to determine its structure and membrane embedment (Koehorst et al. 2004; Nazarov et al. 2007; Vos et al. 2005; Vos et al. 2009; Vos et al. 2007). As a result, the depth of protein insertion, orientation and tilt of the almost straight $\alpha$-helical protein with respect to the bilayer interface are now known with great precision (Nazarov et al. 2007; Vos et al. 2007). These results are in excellent agreement with recent findings from site-directed spin label ESR experiments on the same system (Stopar et al. 2006b). By knowing the membrane-bound structure of M13 coat protein, we now have the challenging opportunity of placing fluorescent polarity and viscosity probes at well-known positions in the protein and at well-known distances with respect to the phospholipid-water bilayer interface to examine the local membrane and/or protein properties. A first step to such an approach is described in our recent paper, where we employed the BADAN label in a steady-state fluorescence study (Koehorst et al. 2008). Since BADAN has a relatively short linker to the protein backbone and shows interesting photophysical properties related to the Stokes shift contributions from water and internal label dynamics, it is a very suitable candidate for such a study.

Previously, we demonstrated that decomposition of the BADAN fluorescence spectrum allows the parameters related to polarity and dynamics to be extracted (Koehorst et al. 2008), e.g., the relative intensity of one spectral component is related to the flexibility of the environment, while the red-shift of another spectral component is related to local polarity. In the present paper, we will focus on the dynamical properties of BADAN-labeled M13 coat protein by using time-resolved fluorescence spectroscopy to provide unprecedented nanoscale profiling of the molecular dynamics and local polarity in protein-lipidwater systems. For this purpose we place BADAN probes covalently attached to membrane-embedded M13 coat protein at well-defined locations inside and outside phospholipid bilayers (see Fig. 1) and use an ultrafast picosecond streak camera setup to measure the BADAN time-resolved fluorescence emission spectra. The location of the BADAN probes in the protein-lipid system is well defined, which arises from the fact that the protein is strongly anchored to the bilayer interface at its C-terminal domain (Stopar et al. 2006a). We take BADAN-labeled mutants with seven key positions from the N-terminal domain to the center of the transmembrane domain, providing locations in the water phase (G03C), close to the headgroup region at the $\mathrm{N}$-terminal protein domain (A07C), just above and below the headgroup/acyl chain interface (A18C and $\mathrm{A} 25 \mathrm{C}$, respectively), in the center of the hydrophobic acyl chain region (V29C and G38C), and at the headgroup/acyl chain interface above the anchoring C-terminal domain (T36C) (Koehorst et al. 2008; Koehorst et al. 2004; Nazarov et al. 2007). In the present study, we use a streak-camera setup to study the BADAN fluorescence and analyze the time-dependent emission spectrum in terms of a two-component excited-state model, related to its planar (PICT) and twisted intramolecular charge transfer (TICT) state. We show that our analysis enables separating contributions by conformational relaxation from the PICT to the TICT state as governed by the label dynamics, hydrogen bonding to local water molecules, and a general solvent relaxation. 


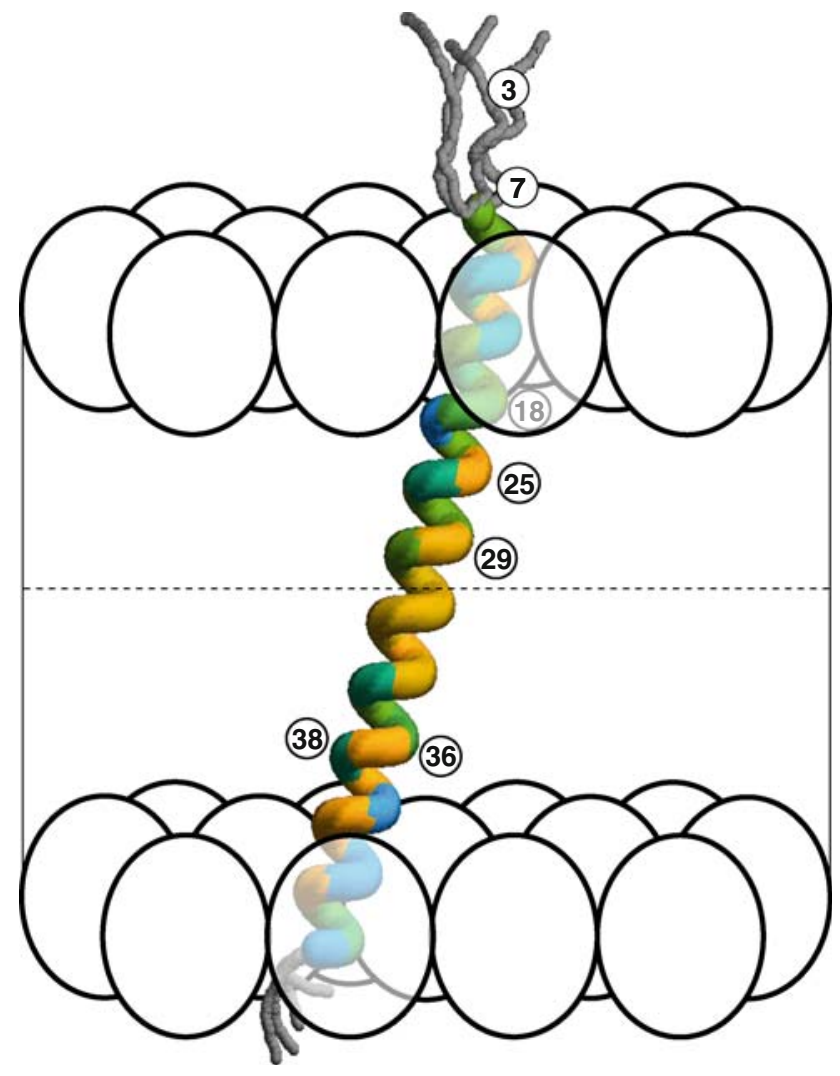

Fig. 1 Location of BADAN-labeled M13 coat protein mutants with respect to the water-membrane interface: in the water phase $(\mathrm{G} 03 \mathrm{C})$, close to the headgroup region at the $\mathrm{N}$-terminal protein domain (A07C), just outside (A18C) and inside (A25C and T36C) the hydrophobic acyl chain region, and in the center of the hydrophobic acyl chain region (V29C and G38C). The ellipses represent the lipid headgroups and the center of the membrane is indicated by a dashed line

\section{Methodology}

Sample preparation

Site-specific cysteine mutants of M13 major coat protein (G03C, A07C, A18C, A25C, V29C, T36C, and G38C) were prepared, purified, and labeled with 6-bromoacetyl2-dimethylaminonaphthalene (BADAN; Invitrogen, Molecular Probes, Carlsbad, CA) in a similar manner to that described previously (Koehorst et al. 2008). BADANlabeled M13 coat protein mutants were reconstituted into phospholipid bilayers as reported earlier (Spruijt et al. 1989). This sample preparation procedure yields small unilamellar vesicles that have a low scattering in the fluorescence decay experiments.

The phospholipid 1,2-dioleoyl-sn-glycero-3-phosphocholine (18:1PC) was purchased from Avanti Polar Lipids (Alabaster, AL) and 1,2-dioleoyl-sn-glycero-3-[phosphorac-(1-glycerol)] (18:1PG) was purchased from Sigma (St. Louis, MO). Mixed lipid bilayers (small unilamellar vesicles with a diameter of $\sim 50 \mathrm{~nm})$ were prepared of 18:1PC and 18:1PG in a 4:1 (mol/mol) ratio, which will be denoted as mixed PC/PG. Highly diluted samples were prepared with a mutant protein concentration of $\sim 1 \mu \mathrm{M}$. The lipid-to-protein ratio of the samples was $\sim 1,500$.

Streak camera fluorescence measurements

For fluorescence decay measurements on a picosecond time scale, mode-locked lasers and a streak camera were used. Reconstituted protein-lipid samples were contained in 1-ml cuvettes. The measuring time for each sample was $20 \mathrm{~min}$. For a detailed description of our streak camera setup, see Van Oort et al. (2009). For the present study, we used vertically polarized $400 \mathrm{~nm}(\sim 200 \mu \mathrm{W})$. The fluorescence signal was passed through a 408-nm long-pass filter (Schott KV 408). The spectrograph was equipped with a turret carrying a ruled grating with 40 grooves $/ \mathrm{mm}$ and blaze wavelength $500 \mathrm{~nm}$. The spectral width was $\sim 320 \mathrm{~nm}$ with the central wavelength set at $500 \mathrm{~nm}$. The time window used was 2,100 ps. The instrument response time was about 3 ps. Scale, linearity, back sweep and curving of the time and wavelength axes were extensively treated in correction procedures (Van Stokkum et al. 2008) using references as Fabry-Perot etalons, fixed wavelength light sources, and a white light reference source (Optronic Laboratories, Orlando, FL model 65A ultra precise current source with model OL 220C $200 \mathrm{~W}$ quartz halogen tungsten lamp). The result is a two-dimensional "image," with time on the vertical and detection wavelength on the horizontal axis.

\section{Reconstruction of artifact-free streak camera images}

The images were corrected for dark current and shading (time and wavelength dependency of the sensitivity) of the camera. Subsequently, the images were fitted by a global analysis procedure (Van Stokkum et al. 2008). In the analysis, four lifetimes and accompanying decay-associated spectra, plus a Raman scatter component, were needed to describe the data. The instrument response function (IRF) was modeled with a Gaussian function. Also the wavelength dependence of time zero (resulting from differences in arrival time of photons of different wavelength) was taken into account in the fit procedure. From the estimated four lifetimes and accompanying decay-associated spectra, so-called artifact-free images on wavenumber scale were reconstructed. The resulting images were analyzed by fitting with time-dependent spectral line shape components using the program IGOR Pro 3.13 (WaveMetrics, Lake Oswego, OR). In the course of this fitting analysis, it was found that excellent robust fits could be obtained by using two line shape functions: (1) a 
symmetric Gaussian line shape, and (2) an asymmetric Gaussian (skewed Gaussian or lognormal line shape) (Burstein and Emelyanenko 1996), having a fixed asymmetry of 0.65 (see section "Results and Discussion" for reasons to choose these line shapes). The decay-associated spectra that emerge from the global analysis reconstruction have regions of negative amplitude on the red side of the emission peak, confirming relaxation processes in our system.

\section{Results and discussion}

Streak camera detected time-resolved fluorescence and image reconstruction

As typical examples, experimental streak images (only corrected for dark current and shading) of BADAN-labeled mutants G03C and V29C are presented in Fig. 2a and b, respectively. By the global analysis procedure described in the previous paragraph, images were all fitted well using four lifetimes. The reconstructed images of mutants G03C and V29C are presented in Fig. 2c and d, respectively. The total time over which the images are reconstructed is $3 \mathrm{~ns}$.
The images clearly show that the fluorescence maximum is shifting in time for a mutant with the BADAN label at the water headgroup interface (mutant G03C, Fig. 2c), while it remains almost constant for a mutant having the label in the center of the hydrophobic acyl chain region (mutant V29C, Fig. 2d). This is related to the fact that BADAN at position 3 is surrounded by dipolar water molecules. These local water molecules can reorient after formation of the excited BADAN state (charge transfer state with a strong dipole moment), thereby lowering its energy. This is called solvent relaxation (SR). In addition, water molecules may also form hydrogen bonds to BADAN, and the BADAN moiety itself may undergo a conformational change from a relatively planar to a relatively twisted excited state. Both hydrogen bonding (Artukhov et al. 2007; Balter et al. 1988; Chapman et al. 1995; Józefowicz et al. 2005; Parasassi and Gratton 1995; Rowe and Neal 2006; Samanta and Fessenden 2000; Viard et al. 1997) and conformational twisting (Abbyad et al. 2007; Lobo and Abelt 2003; Parusel et al. 1998; Vincent et al. 2005) were found to contribute to the relaxation of the fluorescence of the well-known lipid probes PRODAN and LAURDAN, whose chromophore moieties are identical to that of BADAN.
Fig. 2 Experimental (a, b) and reconstructed streak camera images $(\mathbf{c}, \mathbf{d})$ of BADANlabeled M13 coat protein mutants having the label in the polar $(\mathrm{G} 03 \mathrm{C}$, a and $\mathbf{c})$ and hydrophobic core (V29C, b and d) region of mixed $\mathrm{PC} / \mathrm{PG}$ phospholipid bilayers. Note that the horizontal axis of the experimental images is on a wavelength scale $(340-660 \mathrm{~nm})$ and the vertical axis is $2,100 \mathrm{ps}$
A
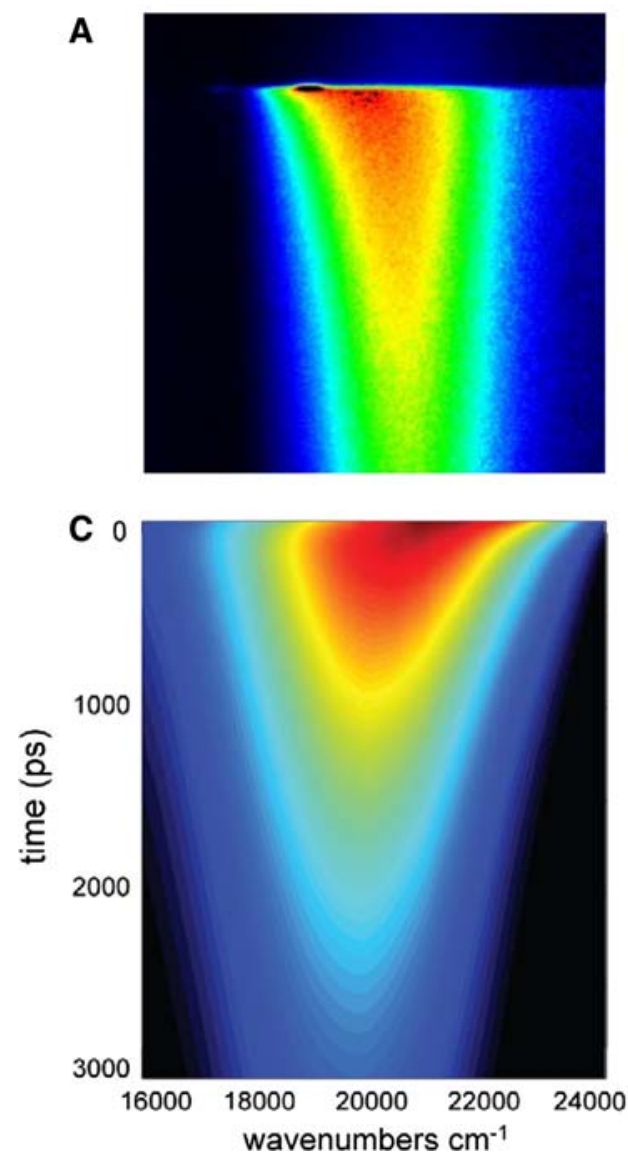

B
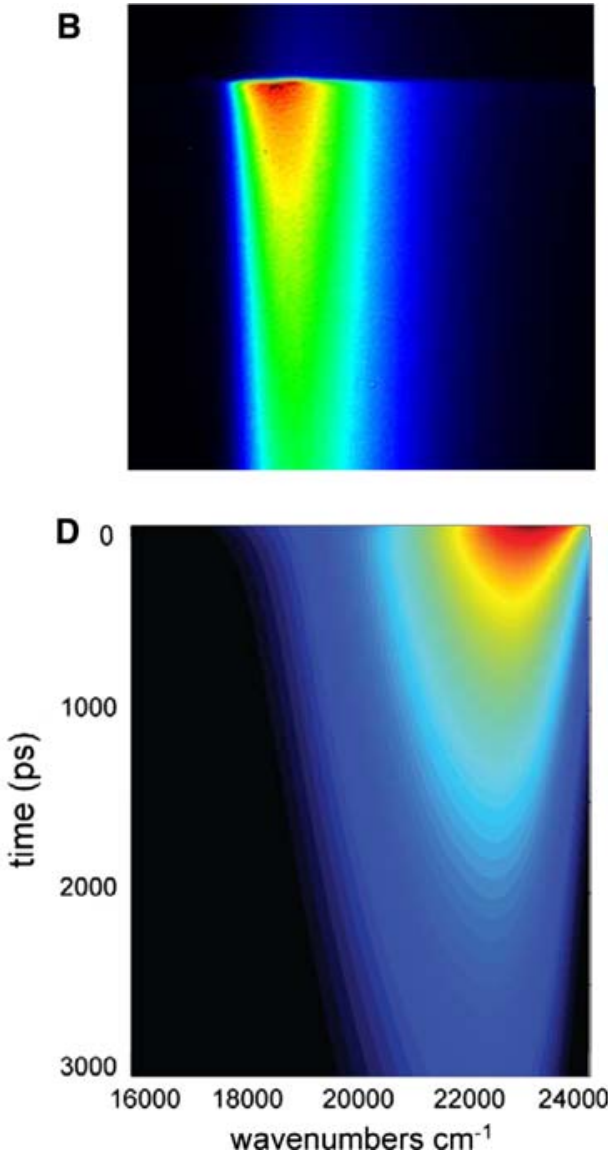
Spectral decomposition of reconstructed images

The reconstructed fluorescence images are further analyzed in terms of time-dependent BADAN fluorescence spectra. In our previous work, steady-state spectra of BADAN were fitted to a set of three Gaussian-shaped components of which two had a fixed spectral position (Koehorst et al. 2008). In this paper we demonstrate that the time-resolved spectra can be fitted well to two components of Gaussian line shape, however, both show time-dependencies of their position. The time-dependency of the intensity of the (relatively) low energy component shows an ingrowth that correlates well with part of the decay of the high-energy component.

For the analysis of the streak images at each time, the fluorescence spectrum is considered to be a superposition of two spectral line shapes: (1) an asymmetric Gaussian (lognormal) line shape associated with an un-relaxed state with relatively weak charge transfer character and (2) a symmetric Gaussian line shape associated with a relaxed state with an increased charge transfer character. The choice of these line shapes is supported by a similar finding for PRODAN fluorescence in heterogeneous environments of different polarity. It was found to consist of two subspectra with different lognormal line shapes. An asymmetric line shape was ascribed to PRODAN in a hydrophobic environment and a symmetric line shape to PRODAN in a nonviscous polar environment (Sachl et al. 2008). Further support for the chosen spectral line shapes comes from fluorescence of tryptophan, an intrinsic polarity probe of proteins. In the small protein mellitin, at a membrane-water interface, the tryptophan fluorescence shows a line shape that gradually becomes more symmetric in time with a clear role of hydration dynamics (Qiu et al. 2005). To avoid problems arising from correlations between the fit parameters, we fixed the asymmetry of the lognormal line shape of the high-energy component and the width of the Gaussian line shape of the low-energy component. The asymmetry of the lognormal line shape was fixed at 0.65 , which is the value found for BADAN attached to mutants with cysteine positions close to the hydrophobic center of the bilayer (i.e., V29C and G38C), where the low-energy component is (almost) absent. The width of the Gaussian line shape was fixed at 2,200 $\mathrm{cm}^{-1}$, which is the value found for BADAN attached to mutants with cysteine positions in the polar water phase (i.e., G03C) after $3 \mathrm{~ns}$ of relaxation. For all mutants, this approach leads to high-quality fits as indicated by the residue plots for the 600-ps spectrum (Fig. 3). The residues for the 30-ps and 3-ns data have a comparable quality.

\section{Label dynamics}

Both the spectral intensities and the positions directly after excitation differ with label position. Furthermore they change as a function of time (Fig. 4). For the PICT state we present the position $\bar{v}_{P 0}$ directly after the excitation pulse, i.e., at $t=1.5 \mathrm{ps}$. Within the time resolution of our streak system, this wavenumber represents the highest unrelaxed energy level of the BADAN label. For the TICT state, $\bar{v}_{T 0}$ is ill-defined for apolar environments of the label because it is absent directly after excitation. Therefore we present the value $\bar{v}_{T 3}$ at $3 \mathrm{~ns}$, representing the lowest BADAN energy level. The intensity of the high-energy component decays bi-exponentially for all label positions, characterized by time constants $\tau_{\mathrm{LD} 1}$ and $\tau_{\mathrm{LD} 2}$ of $100-600$ and 900-2,400 ps, respectively (Fig. 4a; Table 1). This range of relaxation times is in agreement with values found for LAURDAN in reverse micelles (Vincent et al. 2005). For all label positions, the intensity of the low-energy component shows an ingrowth that follows the intensity decay of the high-energy component (Fig. 4b). Therefore the two spectral components can be assigned to a high-energy PICT state and a low-energy TICT state, and the time dependency should arise from a conformational relaxation from the PICT to the TICT state, i.e., by a conformational
Fig. 3 Time-resolved emission spectra obtained from Fig. 2 of BADAN-labeled M13 coat protein mutants having the label in the polar $(\mathrm{G} 03 \mathrm{C}, \mathbf{a})$ and hydrophobic core (V29C, b) region of mixed $\mathrm{PC} / \mathrm{PG}$ phospholipid bilayers. Data and fits are represented by dashed and solid lines, respectively. The spectra are shown at 30 , 600 , and 3,000 ps. For both figures, the residue plots are shown for the 600-ps spectrum
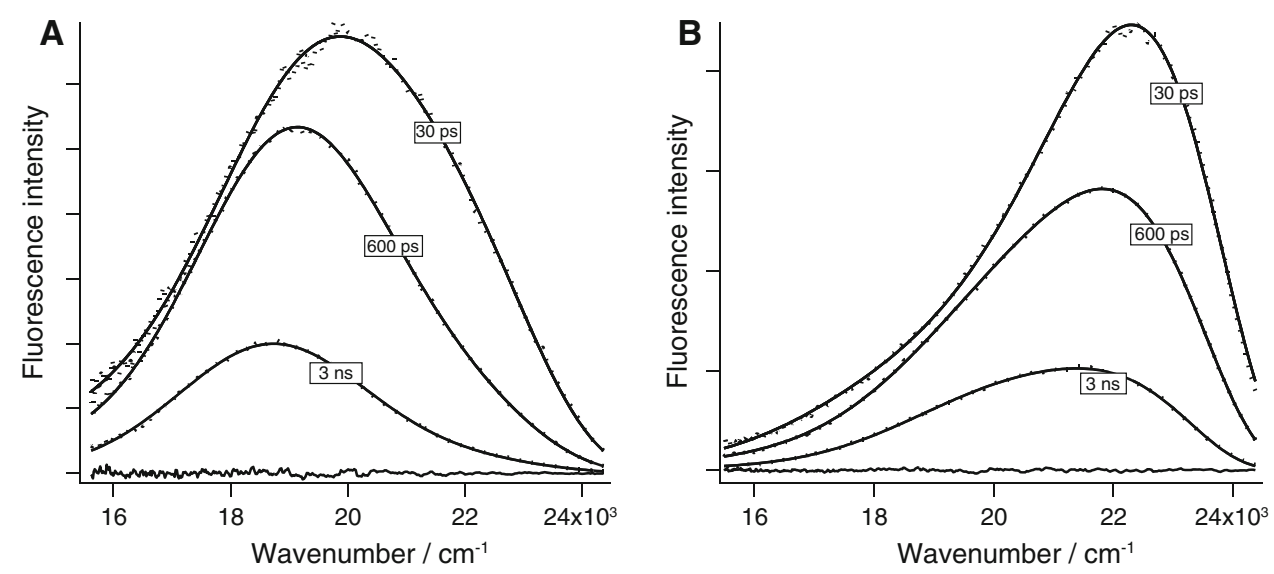
Fig. 4 Analysis of the timeresolved fluorescence spectra of BADAN-labeled M13 coat protein mutants G03C (open symbols) and V29C (closed symbols). The PICT component is indicated in blue and the TICT component in red. For clarity reasons, dashed lines are inserted when data points show large scatter resulting from low intensities of the corresponding spectral component
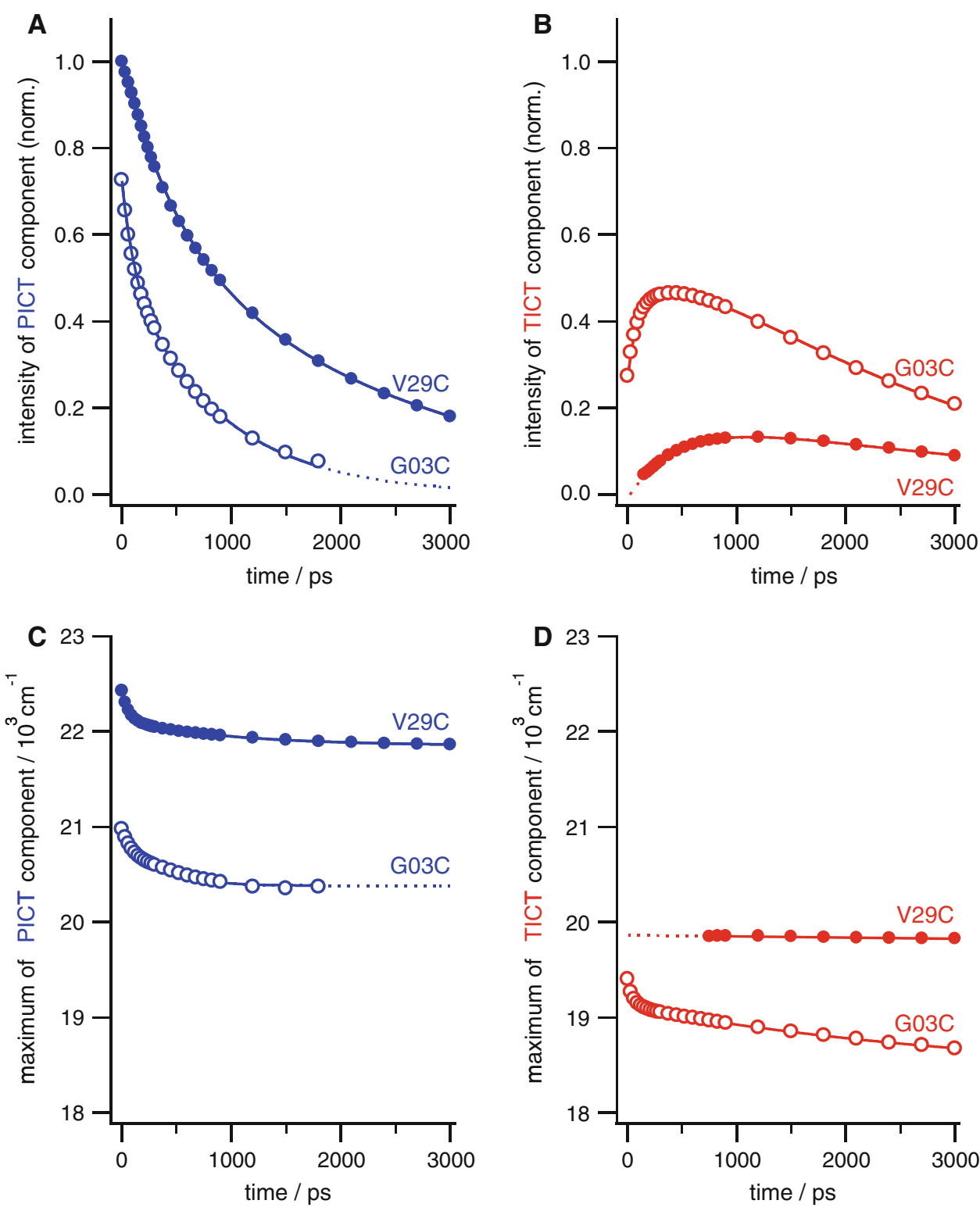

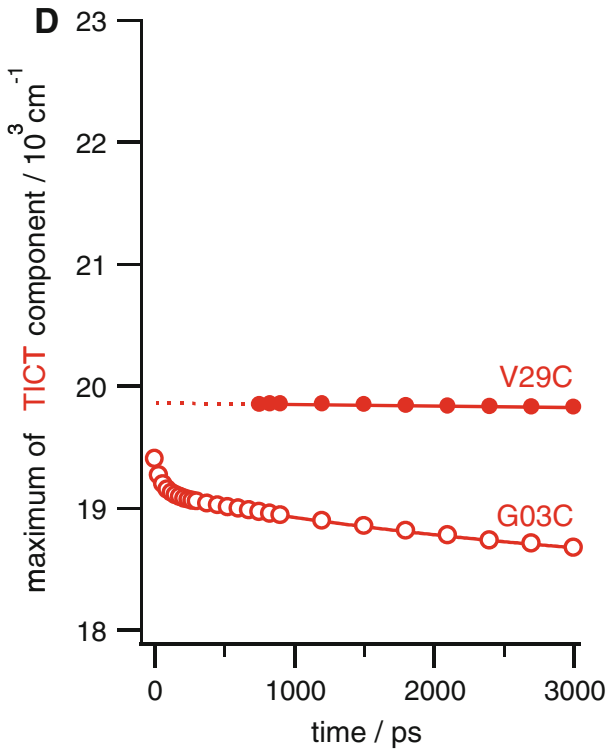

Table 1 Wavenumber positions of the PICT and TICT component $\left(\bar{v}_{P 0}, \bar{v}_{T 3}\right)$, and characteristic time constants of intensity decay $\left(\tau_{\mathrm{LD} 1}, \tau_{\mathrm{LD} 2}\right)$ and wavenumber shift $\left(\tau_{H B}^{P}, \tau_{S R}^{P}, \tau_{H B}^{T}, \tau_{S R}^{S}\right)$ for BADAN-labeled M13 coat protein mutants incorporated in mixed PC/PG phospholipid bilayers

\begin{tabular}{|c|c|c|c|c|c|c|c|c|c|}
\hline \multirow[t]{2}{*}{ Mutant } & \multirow[t]{2}{*}{$D^{\mathrm{a}}(\mathrm{nm})$} & \multicolumn{3}{|l|}{ PICT state } & \multicolumn{3}{|l|}{ TICT state } & \multicolumn{2}{|c|}{ Label dynamics } \\
\hline & & $\bar{v}_{P 0}\left(\mathrm{~cm}^{-1}\right)$ & $\tau_{H B}^{P}(\mathrm{ps})$ & $\tau_{S R}^{P}(\mathrm{~ns})$ & $\bar{v}_{T 3}\left(\mathrm{~cm}^{-1}\right)$ & $\tau_{H B}^{T}(\mathrm{ps})$ & $\tau_{S R}^{T}(\mathrm{~ns})$ & $\tau_{\mathrm{LD} 1}(\mathrm{ps})$ & $\tau_{\mathrm{LD} 2}(\mathrm{~ns})$ \\
\hline G03C & 4.0 & 20,975 & $90(0.35)$ & $0.8(0.65)$ & 18,360 & $50(0.30)$ & $\sim 3(0.70)$ & $110(0.30)$ & $0.9(0.70)$ \\
\hline $\mathrm{A} 07 \mathrm{C}$ & 3.2 & 21,440 & 270 & - & 18,340 & $70(0.15)$ & $0.9(0.85)$ & $150(0.15)$ & $1.5(0.85)$ \\
\hline A18C & 1.7 & 21,300 & $110(0.15)$ & $3.0(0.85)$ & 19,310 & - & 0.7 & $150(0.50)$ & $1.4(0.50)$ \\
\hline $\mathrm{A} 25 \mathrm{C}$ & 0.8 & 22,185 & $80(0.35)$ & $1.7(0.65)$ & 19,925 & $60(0.30)$ & $0.6(0.70)$ & $205(0.15)$ & $2.0(0.85)$ \\
\hline V29C & 0.2 & 22,425 & $70(0.55)$ & $1.1(0.45)$ & 19,825 & - & - & $470(0.45)$ & $2.4(0.55)$ \\
\hline T36C & -0.8 & 22,240 & $80(0.40)$ & $1.5(0.60)$ & 19,115 & $80(0.40)$ & $0.9(0.60)$ & $590(0.45)$ & $2.1(0.55)$ \\
\hline G38C & -0.4 & 22,360 & $80(0.50)$ & $0.9(0.50)$ & 20,650 & $140(0.85)$ & $1.5(0.15)$ & $350(0.25)$ & $2.2(0.75)$ \\
\hline
\end{tabular}

Numbers in parentheses are the relative amplitudes of the corresponding exponentials

${ }^{a}$ Distance to the center of the bilayer 
twisting arising from internal label dynamics (Koehorst et al. 2008). Conformational twisting has been described in the literature as one of the main processes in the fluorescence behavior of the BADAN-related well-known lipid bilayer probes PRODAN and LAURDAN (Abbyad et al. 2007; Lobo and Abelt 2003; Parusel et al. 1998; Vincent et al. 2005).

The results in Table 1 demonstrate that transformation from PICT to TICT state is characterized by two time constants, $\tau_{\mathrm{LD} 1}$ and $\tau_{\mathrm{LD} 2}$. Directly after excitation (1.5 ps), only PICT fluorescence is observed in the hydrophobic core region of the phospholipid bilayer (Fig. 4a, b). However, for label positions in the headgroup region and water phase, the intensity of the TICT component directly after excitation is already $25 \%$. This indicates that in a polar environment part of the BADAN labels is already in a twisted conformation in the ground state, probably facilitated by a favorable surrounding of water molecules. Time constant $\tau_{\mathrm{LD} 1}$ describes the fastest component for the label dynamics; therefore, we assign this time constant to the initial step of the conformational change in the BADAN label.

\section{Hydrogen bonding and water dynamics}

Along with the intensity change, the PICT and TICT components red-shift bi-exponentially in time (Fig. 4c, d). For the PICT state, one of the shifting time constants is in the range from 70 to $110 \mathrm{ps}$ (time constant $\tau_{H B}^{P}$ in Table 1) and the other in the range from 0.8 to $3.0 \mathrm{~ns}$ (time constant $\tau_{S R}^{P}$ in Table 1) for all label positions. For label positions in the hydrophobic interior (i.e., V29C and G38C) the $\sim 100 \mathrm{ps}$ time for the shift of the PICT component is much shorter than the characteristic time involved in conformational twisting, so it must be due to a different process. The excited PICT state of BADAN has a strong dipolar character, leading to a partial negative charge on the oxygen atom of its $\mathrm{C}=\mathrm{O}$ group (Koehorst et al. 2008). Relaxation of this excited state can therefore be facilitated by fast hydrogen bonding to this group of residual water molecules that is present in the vicinity of the BADAN label in the hydrophobic core region of the phospholipid bilayer (Koehorst et al. 2008). In addition to TICT formation, the fluorescence relaxation for PRODAN and LAURDAN probes is also often partly ascribed to specific interactions with the solvent (Artukhov et al. 2007; Balter et al. 1988; Chapman et al. 1995; Józefowicz et al. 2005; Parasassi and Gratton 1995; Samanta and Fessenden 2000; Viard et al. 1997) and the more general solvent relaxation by reorientation of the water molecules after photo-excitation of the probe molecules [see also Koehorst et al. (2008) and references therein].

The shift of the PICT component of V29C and G38C (from $\sim 22,400$ at $1.5 \mathrm{ps}$ to $21,900 \mathrm{~cm}^{-1}$ at $3 \mathrm{~ns}$ ) is in good agreement with our recent results of decomposition of steady-state spectra of the same mutants (Koehorst et al. 2008). For example, fitting of the steady-state spectra with three Gaussian line shapes resulted in dominant contributions of a component at $23,000 \mathrm{~cm}^{-1}$ (ascribed to a nonhydrogen-bonded ICT state) and one at $22,000 \mathrm{~cm}^{-1}$ (ascribed to a hydrogen-bonded ICT state). The timeresolved data of the present study show that these two highenergy steady-state components are a representation of the time-averaged hydrogen bonding in the excited state.

A femtosecond fluorescence study of tryptophan in mellitin at the membrane-water interface revealed three distinct time scales of hydration dynamics (Lu et al. 2004). One short component (0.6-1.3 ps) was said to be close to that of bulk water. The authors assigned a $\sim 9$ ps decay component to hydrogen-bonding water clusters that were in a dynamic exchange with bulk and interfacial water, whereas a $\sim 100$ ps component was assigned to the motion of ordered water molecules. Interestingly, the time range of 70-140 ps for the time constant $\tau_{H B}^{P}$ agrees well with this component, indicating that $\tau_{H B}^{P}$ is related to hydrogen bonding to local, slowed down (ordered) water molecules. In line with this analysis, we assign the initial drop of the energy of the PICT state (with respect to $\bar{v}_{P 0}$ ), which is especially apparent outside the hydrophobic core region of the membrane, to fast relaxation of bulk water molecules. The relatively long ( $>0.8 \mathrm{~ns}$ ) time constant $\tau_{S R}^{P}$ is one order of magnitude slower than these water-related processes. We assign this time constant to solvent relaxation after BADAN excitation due to the slow settlement of distant non-bulk-like water molecules and possibly phospholipid headgroups and polar amino acid residues of the protein. Similar slow processes have been observed in the literature (Lu et al. 2004; Qiu et al. 2005). The time decay of the spectral position of the TICT component has a bi-exponential character. Both time constants $\tau_{H B}^{T}$ and $\tau_{S R}^{T}$ are roughly comparable to the time constants found for hydrogen bonding $\left(\tau_{H B}^{P}\right)$ and solvent relaxation $\left(\tau_{S R}^{P}\right)$ of the PICT state, respectively (Table 1). These findings are summarized in Fig. 5, which describes the photophysics and energy level scheme of the BADAN label.

In a previous study (Koehorst et al. 2008), we presented three states for BADAN: ICT $\left(\sim 23,000 \mathrm{~cm}^{-1}\right.$ arising from non-hydrogen-bonded ICT species), $\mathrm{HICT}_{\mathrm{i}}\left(\sim 22,000 \mathrm{~cm}^{-1}\right.$ arising from hydrogen-bonded BADAN labels in the immobile state), and $\mathrm{HICT}_{\mathrm{m}}\left(19,000-21,000 \mathrm{~cm}^{-1}\right.$ arising from hydrogen-bonded BADAN labels in the mobile state). However, we were unable to assign these states to the PICT and TICT states of BADAN because we were unaware of the underlying dynamical processes, such as conversion of the PICT to the TICT state by label dynamics, solvent relaxation and hydrogen bonding. In the light of the present work, we can improve this assignment by relating the $\mathrm{HICT}_{\mathrm{i}}$ state to 


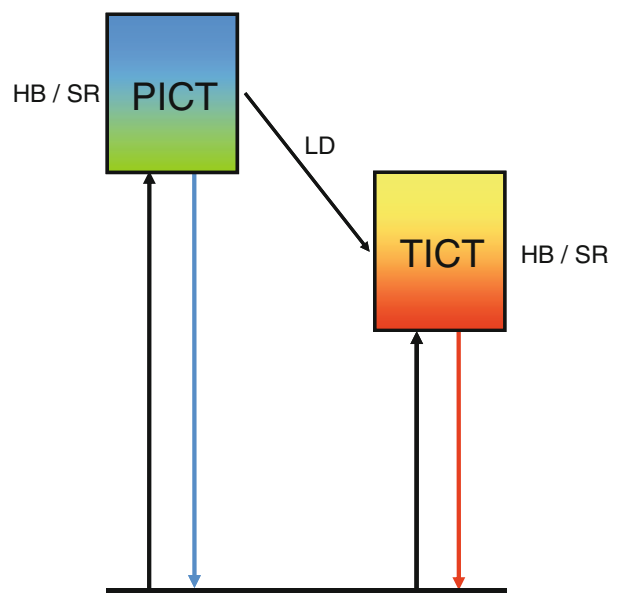

Fig. 5 Energy level scheme of the BADAN label, illustrating the relative energies of the two excited states (i.e., PICT and TICT), the transition from PICT to TICT (by label dynamics, $L D$ ), and relaxation (indicated by color shading) of both states by the solvent in a general way $(S R)$ and via specific interactions (i.e., hydrogen bonding, $H B$ ). The related characteristic time constants for these processes are presented in Table 1. The black arrows indicate direct BADAN excitation to the PICT and TICT state. The blue and red arrows represent the fluorescence from the PICT and TICT states, respectively. The fluorescence decay time for the TICT state is approximately $3 \mathrm{~ns}$, as found from time-correlated single-photon counting experiments of BADAN-labeled M13 coat protein mutants incorporated in mixed PC/PG phospholipid bilayers (data not shown)

sterically inflexible BADAN in the PICT state, being unable to convert to the TICT state by label dynamics. On short time scales, H-bonding has already taken place, so in the steadystate fluorescent experiment, all these BADAN labels can be considered to be hydrogen bonded. A similar assignment can be made for the $\mathrm{HICT}_{\mathrm{m}}$ state, which is a reservoir of BADAN labels that are directly excited in the TICT state as well TICT states that have originated from conversion of the PICT state by label dynamics. Again, also for the TICT state, H-bonding has already taken place on a short time scale. In our present analysis, we have neglected the ICT state for the following reasons: (1) In most cases, the ICT state has a small contribution because of the presence of water molecules at almost all positions of the BADAN label. (2) Including the ICT state in the model would have introduced more fit parameters and an increased correlation among the fitting parameters, which was undesired. (3) In the present work, we excited the sample at $400 \mathrm{~nm}$, in contrast to the steady-state fluorescent data that were obtained at an excitation of $380 \mathrm{~nm}$. At this higher wavelength, fewer ICT states are excited because of the red edge effect.

\section{Membrane profiling}

The location and tilt of the M13 coat protein in the phospholipid bilayer is known (Koehorst et al. 2004). Therefore we can calculate the position $\mathrm{D}$ of the
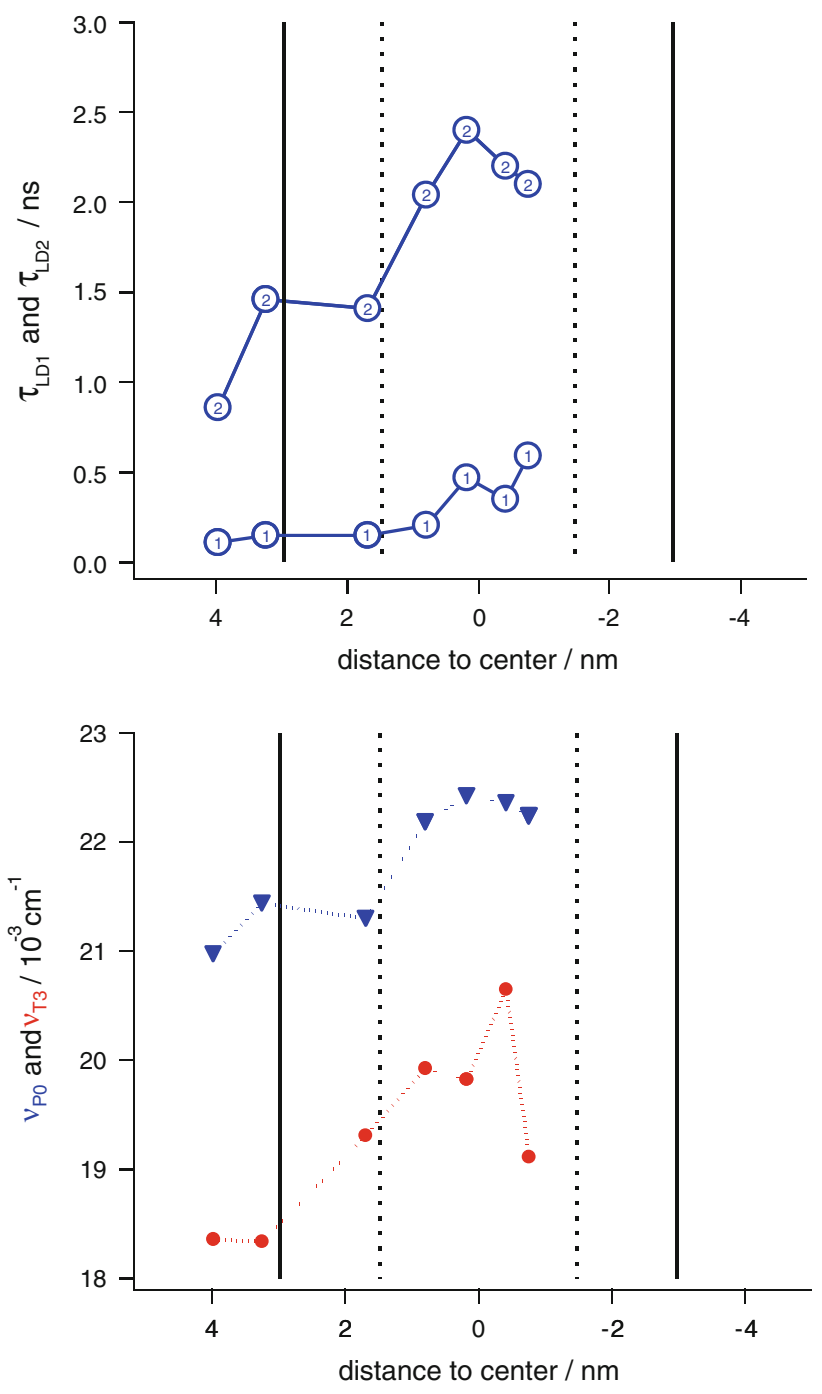

Fig. 6 Membrane profiles of $\tau_{\mathrm{LD} 1}$ and $\tau_{\mathrm{LD} 2}$ (upper panel) related to the microviscosity of the bilayer and profiles of $\bar{v}_{P 0}$ and $\bar{v}_{T 3}$ (lower panel) originating from differences in local water concentration and related polarity

BADAN label with respect to the center of the bilayer (Table 1). This allows nanoscale profiling of the BADAN parameters through the membrane. Note that as a result of the tilt of the helix, the label at position 36 is closer to the interfacial region than that at position 38 . Within experimental error, time constants $\tau_{H B}^{P}, \tau_{S R}^{P}, \tau_{H B}^{T}$, and $\tau_{S R}^{T}$ do not show a clear profile over the bilayer (Table 1), probably because our analysis model has a too limited sensitivity for these parameters. However, the other parameters are clearly dependent on the bilayer position of the BADAN label (Fig. 6). The spectral positions of the PICT and TICT states increase on entering the hydrophobic core of the membrane. This indicates that fewer water molecules are available for relaxing these states, which is a measure for the local water concentration and related polarity. This finding is 
in agreement with fluorescence data obtained with fluorescent dye in phospholipid systems, located at various depths in the bilayer (Hutterer et al. 1996; Jurkiewicz et al. 2006; Sýkora et al. 2007). On the other hand, the label dynamics slows down on entering the headgroup region of the membrane (increase of $\tau_{\mathrm{LD} 1,2}$ ). The dynamics further slow down on entering the hydrophobic core of the membrane. This profile is related to the local microviscosity of the membrane system (Blatt and Sawyer 1985). As such, our approach provides an unprecedented nanoscale profiling of the dynamics and distribution of water in membrane systems. The strongest effects are found for the parameters $\bar{v}_{P 0}$ and $\tau_{\mathrm{LD} 2}$, and it can be seen that, especially at the region of the carbonyl groups of the acyl chains, these parameters show a large change. This provides clear evidence that the actual barrier of membranes for ions and aqueous solvents is located at this membrane region.

In our experiments M13 coat protein is used only as a scaffolding for the BADAN label, and the results are interpreted in terms of lipid-water bilayer dynamics. However, as a word of caution, it should be noted that any introduction of a fluorescent label can influence the system studied. However, recent work on BADANlabeled WALP peptides introduced in phospholipid bilayers shows that the disturbing effect of BADAN is small (Holt et al. 2009). Nevertheless, the introduction of the transmembrane M13 coat protein may also alter the properties of the phospholipid bilayer. Therefore, a generalization of our results to lipid-water bilayers in general should be made with care.

\section{Conclusion}

In conclusion, we have shown that spectral decomposition of the time-resolved fluorescence data provides the characteristic time constant for the twisting motion of the BADAN label, which is sensitive to the local flexibility of the protein-lipid environment. In addition, our analysis provides information about the mobility of the water molecules bound to the membrane, given by the hydrogen bonding. By knowing the precise location of the BADAN label attached to a well-anchored membrane protein, such as the M13 coat protein, these parameters can be mapped on a nanoscale throughout the membrane. In addition, this approach will provide new opportunities to obtain local molecular information about water molecules in heterogeneous biosystems.

Acknowledgments This work is part of the research program of the "Stichting voor Fundamenteel onderzoek der Materie (FOM)", which is financially supported by the "Nederlandse Organisatie voor Wetenschappelijk Onderzoek (NWO)." B.V.O. was supported by FOM.
Open Access This article is distributed under the terms of the Creative Commons Attribution Noncommercial License which permits any noncommercial use, distribution, and reproduction in any medium, provided the original author(s) and source are credited.

\section{References}

Abbyad P, Shi X, Childs W, McAnaney TB, Cohen BE, Boxer SG (2007) Measurement of solvation responses at multiple sites in a globular protein. J Phys Chem B 111:8269-8276

Artukhov VY, Zharkova OM, Morozova JP (2007) Features of absorption and fluorescence spectra of prodan. Spectrochim Acta Pt A-Molec Biomolec Spectrosc 68:36-42

Balter A, Nowak W, Pawelkiewicz W, Kowalczyk A (1988) Some remarks on the interpretation of the spectral properties of prodan. Chem Phys Lett 143:565-570

Blatt E, Sawyer WH (1985) Depth-dependent flourescent quenching in micelles and membranes. Biochim Biophys Acta 822:43-62

Burstein EA, Emelyanenko VI (1996) Log-normal description of fluorescence spectra of organic fluorophores. Photochem Photobiol 64:316-320

Chapman CF, Fee RS, Maroncelli M (1995) Measurements of the solute dependence of solvation dynamics in 1-propanol: the role of specific hydrogen-bonding interactions. J Phys Chem 99:4811-4819

Ebbinghaus S, Kim SJ, Heyden M, Yu X, Heugen U, Gruebele M, Leitner DM, Havenith M (2007) An extended dynamical hydration shell around proteins. Proc Natl Acad Sci USA 104:20749-20752

Ebbinghaus S, Kim SJ, Heyden M, Yu X, Gruebele M, Leitner DM, Havenith M (2008) Protein sequence- and pH-dependent hydration probed by terahertz spectroscopy. J Am Chem Soc 130:2374-2375

Epand RM, Kraayenhof R (1999) Fluorescent probes used to monitor membrane interfacial polarity. Chem Phys Lipids 101:57-64

Halle B (2004) Protein hydration dynamics in solution: a critical survey. Philos Trans R Soc B-Biol Sci 359:1207-1224

Holt A, Koehorst RBM, Rutters-Meijneke T, Gelb MH, Rijkers DTS, Hemminga MA, Killian JA (2009) Tilt and rotation angles of a transmembrane model peptide as studied by fluorescence spectroscopy. Biophys J (in press)

Hutterer R, Schneider FW, Sprinz H, Hof M (1996) Binding and relaxation behaviour of prodan and patman in phospholipid vesicles: a fluorescence and ${ }^{1} \mathrm{H}$ NMR study. Biophys Chem 61:151-160

Jasnin M, Moulin M, Haertlein M, Zaccai G, Tehei M (2008) Down to atomic-scale intracellular water dynamics. EMBO Rep 9:543547

Józefowicz M, Kozyra KA, Heldt JR, Heldt J (2005) Effect of hydrogen bonding on the intramolecular charge transfer fluorescence of 6-dodecanoyl-2-dimethylaminonaphtalene. Chem Phys 320:45-53

Jurkiewicz P, Olzyńska A, Langner M, Hof M (2006) Headgroup hydration and mobility of DOTAP/DOPC bilayers: a fluorescence solvent relaxation study. Langmuir 22:8741-8749

Klymchenko AS, Mély Y, Demchenko AP, Duportail G (2004) Simultaneous probing of hydration and polarity of lipid bilayers with 3-hydroxyflavone fluorescent dyes. Biochim Biophys Acta 1665:6-19

Koehorst RBM, Spruijt RB, Vergeldt FJ, Hemminga MA (2004) Lipid bilayer topology of the transmembrane $\alpha$-helix of M13 major coat protein and bilayer polarity profile by site-directed fluorescence spectroscopy. Biophys J 87:1445-1455 
Koehorst RBM, Spruijt RB, Hemminga MA (2008) Site-directed fluorescence labeling of a membrane protein with BADAN: probing protein topology and local environment. Biophys $\mathrm{J}$ 94:3945-3955

Lakowicz JR (2006) Principles of fluorescence spectroscopy, 3rd edn. Springer, New York

Lobo BC, Abelt CJ (2003) Does PRODAN possess a planar or twisted charge-transfer excited state? Photophysical properties of two PRODAN derivatives. J Phys Chem A 107:10938-10943

Lu W, Kim J, Qiu W, Zhong D (2004) Femtosecond studies of tryptophan solvation: correlation function and water dynamics at lipid surfaces. Chem Phys Lett 388:120-126

Nazarov PV, Koehorst RBM, Vos WL, Apanasovich VV, Hemminga MA (2007) FRET study of membrane proteins: determination of the tilt and orientation of the N-terminal domain of M13 major coat protein. Biophys J 92:1296-1305

Oleinikova A, Smolin N, Brovchenko I (2007) Influence of water clustering on the dynamics of hydration water at the surface of a lysozyme. Biophys J 93:2986-3000

Parasassi T, Gratton E (1995) Membrane lipid domains and dynamics as detected by Laurdan fluorescence. J Fluoresc 5:59-69

Parusel ABJ, Nowak W, Grimme S, Kohler G (1998) Comparative theoretical study on charge-transfer fluorescence probes: 6-propanoyl-2-( $N, N$-dimethylamino) naphthalene and derivatives. J Phys Chem A 102:7149-7156

Persson E, Halle B (2008) Cell water dynamics on multiple time scales. Proc Natl Acad Sci USA 105:6266-6271

Qiu W, Zhang L, Kao Y-T, Lu W, Li T, Kim J, Sollenberger GM, Wang L, Zhong D (2005) Ultrafast hydration dynamics in melittin folding and aggregation: helix formation and tetramer self-assembly. J Phys Chem B 109:16901-16910

Rowe BA, Neal SL (2006) Photokinetic analysis of PRODAN and LAURDAN in large unilamellar vesicles from multivariate frequency-domain fluorescence. J Phys Chem B 110:1502115028

Sachl R, Stepanek M, Prochazka K, Humpolickova J, Hof M (2008) Fluorescence study of the solvation of fluorescent probes prodan and laurdan in poly(e-caprolactone)-block-poly(ethylene oxide) vesicles in aqueous solutions with tetrahydrofurane. Langmuir 24:288-295

Samanta A, Fessenden RW (2000) Excited state dipole moment of PRODAN as determined from transient dielectric loss measurements. J Phys Chem A 104:8972-8975

Spruijt RB, Wolfs CJAM, Hemminga MA (1989) Aggregation-related conformational change of the membrane-associated coat protein of bacteriophage M13. Biochemistry 28:9158-9165
Stopar D, Spruijt RB, Hemminga MA (2006a) Anchoring mechanisms of membrane-associated M13 major coat protein. Chem Phys Lipids 141:83-93

Stopar D, Strancar J, Spruijt RB, Hemminga MA (2006b) Motional restrictions of membrane proteins: a site-directed spin labeling study. Biophys J 91:3341-3348

Sýkora J, Slavicek P, Jungwirth P, Barucha J, Hof M (2007) Timedependent stokes shifts of fluorescent dyes in the hydrophobic backbone region of a phospholipid bilayer: combination of fluorescence spectroscopy and ab initio calculations. J Phys Chem B 111:5869-5877

Van Oort B, Murali S, Wientjes E, Koehorst RBM, Spruijt RB, van Hoek A, Croce R, van Amerongen H (2009) Ultrafast resonance energy transfer from a site-specifically attached fluorescent chromophore reveals the folding of the N-terminal domain of CP29. Chem Phys 357:113-119

Van Stokkum IHM, van Oort B, van Mourik F, Gobets B, van Amerongen H (2008) (Sub)-picosecond spectral evolution of fluorescence studied with a synchroscan streak-camera system and target analysis. In: Aartsma TJ, Matysik J (eds) Biophysical techniques in photosynthesis, vol 26. Springer, Dordrecht, pp 223-240

Viard M, Gallay J, Vincent M, Meyer O, Robert B, Paternostre M (1997) Laurdan solvatochromism: solvent dielectric relaxation and intramolecular excited-state reaction. Biophys J 73:2221-2234

Vincent M, de Foresta B, Gallay J (2005) Nanosecond dynamics of a mimicked membrane-water interface observed by time-resolved stokes shift of LAURDAN. Biophys J 88:4337-4350

Vos WL, Koehorst RBM, Spruijt RB, Hemminga MA (2005) Membrane-bound conformation of M13 major coat protein: a structure validation through FRET-derived constraints. J Biol Chem 280:38522-38527

Vos WL, Schor M, Nazarov PV, Koehorst RBM, Spruijt RB, Hemminga MA (2007) Structure of membrane-embedded M13 major coat protein is insensitive to hydrophobic stress. Biophys $\mathbf{J}$ 93:3541-3547

Vos WL, Nazarov PV, Koehorst RBM, Spruijt RB, Hemminga MA (2009) From 'I' to 'L' and back again: the odyssey of membrane-bound M13 protein. Trends Biochem Sci 34:249-255

Wood K, Plazanet M, Gabel F, Kessler B, Oesterhelt D, Tobias DJ, Zaccai G, Weik M (2007) Coupling of protein and hydrationwater dynamics in biological membranes. Proc Natl Acad Sci USA 104:18049-18054

Zhang L, Wang L, Kao Y-T, Qiu W, Yang Y, Okobiah O, Zhong D (2007) Mapping hydration dynamics around a protein surface. Proc Natl Acad Sci USA 104:18461-18466 